\title{
Spontaneous Intracranial Hypotension and a successful epidural blood patch: Case Report
}

Margarida Candeias, Bernardo Ribeiro, Ana Isabel André

Centro Hospitalar de Lisboa Ocidental, Dept of Anaesthesiology, Lisbon, Portugal

Head of department - Manuel Chedas

\section{Introduction}

Spontaneous Intracranial Hypotension is secondary to a loss of cerebrospinal fluid (CSF) volume which, in turn, is usually caused by spontaneous CSF leaks ${ }^{1}$. Although this uncommon entity has a common presentation, its diagnosis, evaluation and treatment ${ }^{2}$ are challenging.

\section{Case report}

$>$ A 39-year-old woman developed orthostatic headaches, pain in the posterior triangle of the neck and shoulders, as well as nausea and vomiting, without focal signal abnormalities;

$>$ Her medical history includes hypothyroidism and two epidural anaesthesias prior to caesarean sections (2008 and 2015);

$>$ Pain treatment consisting of conservative measures, bed rest, $500 / 50 \mathrm{mg}$ of paracetamol/caffeine $8 / 8$ hours, $250 \mathrm{mg}$ of naproxen $12 / 12$ hours and $4 \mathrm{mg}$ of thiocolchicoside $8 / 8$ hours was ineffective and meningitis was ruled out after a lumbar puncture;

$>$ A head MRI with intravenous injection of paramagnetic contrast material showed bilateral frontoparietal dural signal enforcement correlated with CSF hypotension. A spine CT scan combined with a myelography showed a meningeal leaking at the level of D3;

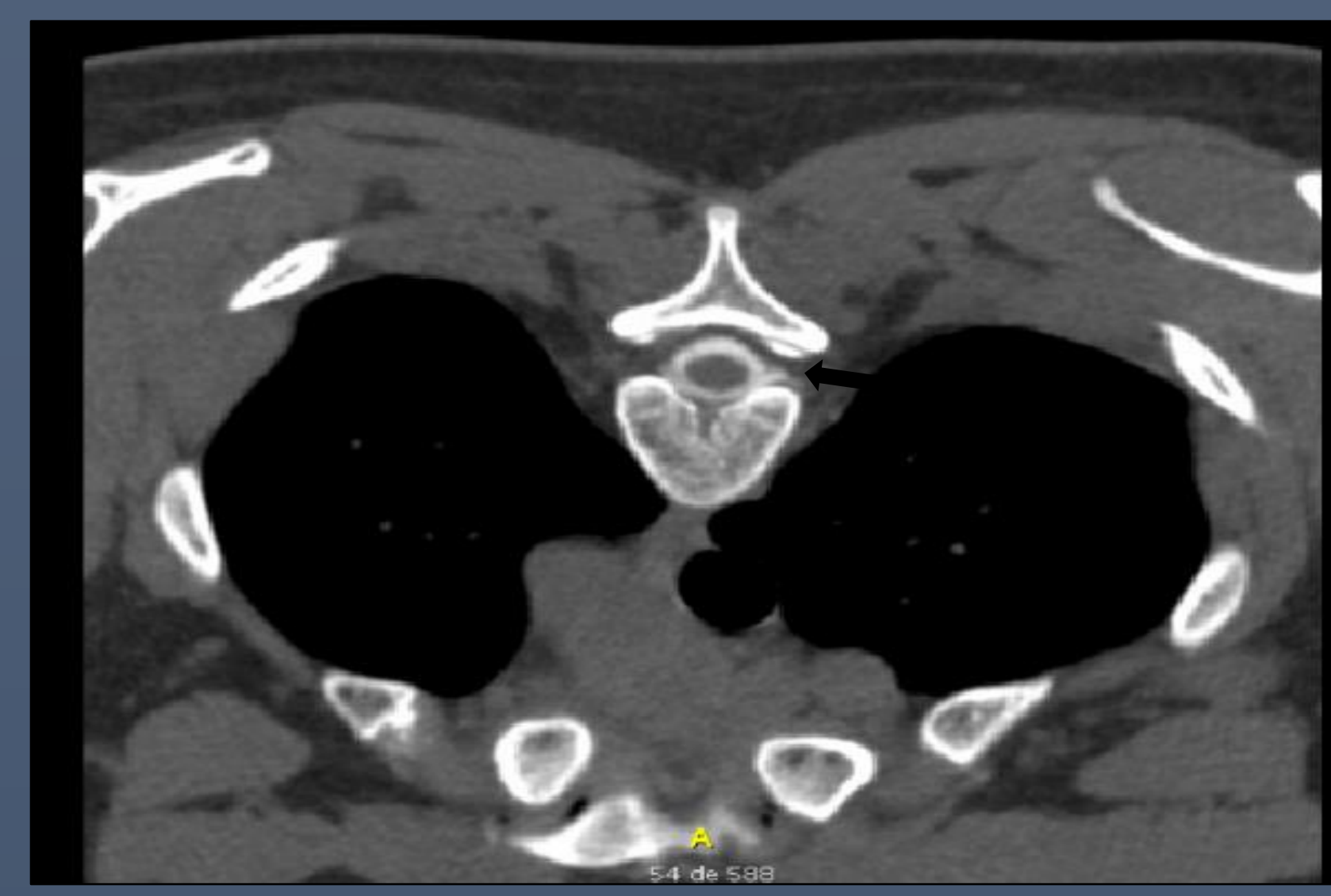

With anesthesia's collaboration, neurosurgery decided the epidural blood patch would be performed in the OR under aseptic technique and with radiologic guidance to identify the space between D6D7. An epidural space puncture was performed with a G18 Tuohy needle, with loss of resistance at $4,5 \mathrm{~cm}$ and $18 \mathrm{ml}$ of homologous blood were injected epidurally;

The patient had an uneventful recovery and was discharged one week later without any symptoms.

\section{Discussion}

The anatomy of a spontaneous CSF leak is frequently complex. CT myelography is the study of choice ${ }^{1}$ to locate the site of the leak and an epidural blood patch is the main and most effective treatment. 